\title{
De novo translation initiation on membrane-bound ribosomes as a mechanism for localization of cytosolic protein mRNAs to the endoplasmic reticulum
}

\author{
SUJATHA JAGANNATHAN, ${ }^{1}$ DAVID W. REID, ${ }^{2}$ AMANDA H. COX, ${ }^{2}$ and CHRISTOPHER V. NICCHITTA ${ }^{1,2}$ \\ ${ }^{1}$ Department of Cell Biology, ${ }^{2}$ Department of Biochemistry, Duke University Medical Center, Durham, North Carolina 27710, USA
}

\begin{abstract}
The specialized protein synthesis functions of the cytosol and endoplasmic reticulum compartments are conferred by the signal recognition particle (SRP) pathway, which directs the cotranslational trafficking of signal sequence-encoding mRNAs from the cytosol to the endoplasmic reticulum (ER). Although subcellular mRNA distributions largely mirror the binary pattern predicted by the SRP pathway model, studies in mammalian cells, yeast, and Drosophila have also demonstrated that cytosolic protein-encoding mRNAs are broadly represented on ER-bound ribosomes. A mechanism for such noncanonical mRNA localization remains, however, to be identified. Here, we examine the hypothesis that de novo translation initiation on ERbound ribosomes serves as a mechanism for localizing cytosolic protein-encoding mRNAs to the ER. As a test of this hypothesis, we performed single molecule RNA fluorescence in situ hybridization studies of subcellular mRNA distributions and report that a substantial fraction of mRNAs encoding the cytosolic protein GAPDH resides in close proximity to the ER. Consistent with these data, analyses of subcellular mRNA and ribosome distributions in multiple cell lines demonstrated that cytosolic protein mRNA-ribosome distributions were strongly correlated, whereas signal sequence-encoding mRNA-ribosome distributions were divergent. Ribosome footprinting studies of ER-bound polysomes revealed a substantial initiation codon read density enrichment for cytosolic protein-encoding mRNAs. We also demonstrate that eukaryotic initiation factor $2 \alpha$ is bound to the ER via a salt-sensitive, ribosome-independent mechanism. Combined, these data support ER-localized translation initiation as a mechanism for mRNA recruitment to the ER.
\end{abstract}

Keywords: mRNA; endoplasmic reticulum; translation initiation; ribosome; mRNA localization; signal sequence

\section{INTRODUCTION}

mRNA localization is an important mechanism for the spatial control of protein synthesis. Prominently studied examples of mRNA localization, notably embryonic polarity determination in the Drosophila embryo, directed cell migration, and neurite morphogenesis, have revealed a common mechanism where cis-encoded localization information (zip codes), cognate RNA binding proteins, and molecular motors direct translationally silenced mRNAs to specific subcellular locales (Martin and Ephrussi 2009; Eliscovich et al. 2013). In addition to these cell-specific mRNA localization phenomena, all eukaryotic cells localize signal peptide-encoding mRNAs to the endoplasmic reticulum (ER) by a distinct, translationdependent mechanism, the signal recognition particle (SRP) pathway (Lingappa and Blobel 1980; Walter and Johnson 1994; Akopian et al. 2013). In the SRP pathway, cytosolic ribo-

Corresponding author: christopher.nicchitta@duke.edu

Article published online ahead of print. Article and publication date are at http://www.rnajournal.org/cgi/doi/10.1261/rna.045526.114. somes engaged in the translation of signal peptide-encoding mRNAs are recognized early in translation via binding of the SRP to the nascent signal peptide, and the mRNA/ribosome/nascent chain (RNC) complex is then localized to the ER via SRP/SRP receptor interactions (Walter and Blobel 1981a,b; Walter et al. 1981). At the ER, direct binding interactions between the translating ribosome and the Sec61 translocon enable cotranslational protein translocation and serve to anchor the localized mRNA to the ER (Walter and Johnson 1994).

While the role of the SRP pathway in mRNA/protein sorting to the ER is well established, substantial evidence for additional mechanisms of mRNA localization to the ER has been reported (Diehn et al. 2000; Lerner et al. 2003; Nicchitta et al. 2005; Loya et al. 2008; Pyhtila et al. 2008; Cui et al. 2012;

(c) 2014 Jagannathan et al. This article is distributed exclusively by the RNA Society for the first 12 months after the full-issue publication date (see http:// rnajournal.cshlp.org/site/misc/terms.xhtml). After 12 months, it is available under a Creative Commons License (Attribution-NonCommercial 4.0 International), as described at http://creativecommons.org/licenses/by-nc/4.0/. 
Kraut-Cohen et al. 2013). Of particular interest, studies conducted in both cells and tissues have demonstrated that cytosolic protein-encoding mRNAs are broadly represented on the ER (Mechler and Rabbitts 1981; Mueckler and Pitot 1981; Diehn et al. 2000; Lerner et al. 2003; Chen et al. 2011; Reid and Nicchitta 2012). It appears, then, that in addition to pathways for directing signal sequence-encoding mRNAs to the ER, there also exists a mechanism(s), as yet unknown, which enables the translation of virtually the entire cytosolic protein-encoding mRNA transcriptome on the ER. One possible mechanism for the broad representation of cytosolic protein-encoding mRNAs on the ER comes from earlier observations that ER-associated ribosomes can initiate translation de novo and in this process are not selective for signal peptide-encoding mRNAs (Potter and Nicchitta 2002). These findings raise the possibility that, if ER-bound ribosomes are initiation-competent in vivo and do not select for signal peptide-encoding mRNAs, then cytosolic proteinencoding mRNAs could undergo translation on ER-bound ribosomes. In addition, if both cytosolic and ER-bound ribosomes have similar capacities for de novo initiation, the subcellular distribution of cytosolic protein-encoding mRNAs would be expected to mirror the steady-state subcellular ribosome distribution.

Here, we demonstrate that cytosolic protein-encoding RNAs $\left(\mathrm{mRNA}_{\text {cyt }}\right)$ can, indeed, be ER-localized and that the extent of ER localization correlates with the fraction of ribosomes that are ER-bound in a given cell at a given time. To determine if ER-bound ribosomes can initiate translation in vivo, we utilized compartment-specific ribosome footprinting/RNA-Seq data (Reid and Nicchitta 2012) to map ribosome distributions on cytosolic protein-encoding mRNAs in the ER and cytosol compartments and report that the mRNA translational profiles in the two compartments are very similar and include a substantial read enrichment at start codons. In addition, we demonstrate that a substantial fraction of the key initiation factor eIF $2 \alpha$ is associated with the ER, independently of bound ribosomes. Taken together, these data support translation initiation as a mechanism for the localization of cytosolic mRNAs to the ER and further illustrate that ER-localized translation serves a prominent and perhaps primary role in the expression of the mRNA transcriptome.

\section{RESULTS}

\section{Cytosolic protein-encoding mRNAs and ribosomes display parallel subcellular distributions}

Past studies conducted in tissue culture cells and tissue-derived subcellular fractions have identified cytosolic proteinencoding mRNAs on the endoplasmic reticulum (Mechler and Rabbitts 1981; Mueckler and Pitot 1981; Diehn et al. 2000; Lerner et al. 2003; Chen et al. 2011; Reid and Nicchitta 2012). Lacking encoded signal sequences, however, the mech- anism of cytosolic protein-encoding mRNA localization to the ER remains enigmatic and, to a degree, controversial (del Alamo et al. 2011). Recent ribosome footprinting studies demonstrate that essentially all cytosolic protein-encoding mRNAs are represented on membrane-bound polysomes, though enriched on cytosolic polysomes (Reid and Nicchitta 2012). We, therefore, considered general, translation-linked mechanisms that might enable the translation of mRNA $_{\text {cyt }}$ on the ER. One such mechanism could be translation initiation, if both cytosolic and ER-bound ribosomes are competent for de novo translation irrespective of the trafficking fate of the encoded polypeptide. In this view, the SRP pathway would direct the localization of signal sequence-encoding mRNAs $\left(\mathrm{mRNA}_{\mathrm{sec}}\right)$ from the cytosol to the ER, as has been established, and the $\mathrm{mRNA}_{\text {cyt }}$ cohort would be translated on both cytosolic and ER-bound ribosomes, to yield the overlapping subcellular distributions noted above.

As an experimental approach to testing this model, we employed a sequential detergent fractionation method to separate cytosolic and ER-associated polysomes. In this method, tissue culture cells are first treated with low concentrations of digitonin to selectively solubilize the plasma membrane and release cytosolic mRNAs/ribosomes, and ER-associated mRNAs/ribosomes are subsequently extracted from the digitonin-treated cells with the nonionic detergent dodecylmaltoside (DDM) (Stephens et al. 2008; Jagannathan et al. 2011). This fractionation method, as applied to HeLa cells, is illustrated in Figure 1A,B. Immunoblot analysis of subcellular fractions (Fig. 1A) shows that the cytosolic marker proteins GAPDH and (monomeric) tubulin are highly enriched in the cytosol fraction, and the resident ER proteins TRAPa, a membrane protein, and GRP94, a lumenal protein, are entirely present in the ER fraction. In companion experiments, HeLa cells were immunostained for tubulin and TRAPa at each stage of the fractionation sequence. As shown in Figure 1B, tubulin staining is entirely lost following digitonin treatment, though TRAPa staining remains similar to control cells. Subsequent treatment of the cells with DDM yields the efficient solubilization of the ER and loss of TRAPa staining.

We next visualized the subcellular distribution of representative mRNAs by single molecule RNA fluorescence in situ hybridization (smRNA-FISH). smRNA-FISH renders individual mRNA molecules visible as diffraction-limited spots (Raj et al. 2008). However, and because of the resolution limits of conventional optical imaging, the smRNAFISH approach was combined with sequential detergent fractionation and ER protein immunostaining, to better distinguish cytosolic- vs. ER-localized mRNAs. The micrographs illustrated in Figure 1C detail typical subcellular distribution profiles of a proxy mRNA $\mathrm{m}_{\text {cyt }}, \mathrm{GAPDH}$, and $\mathrm{mRNA}_{\text {sec }}$, GRP94, with respect to the ER network (TRAPa) of unfractionated cells. Whereas GAPDH mRNAs are distributed throughout the cell, overlapping with the ER though with an apparent enrichment toward the cell periphery, GRP94 mRNAs reside almost entirely in close proximity to the ER membrane. To 
A
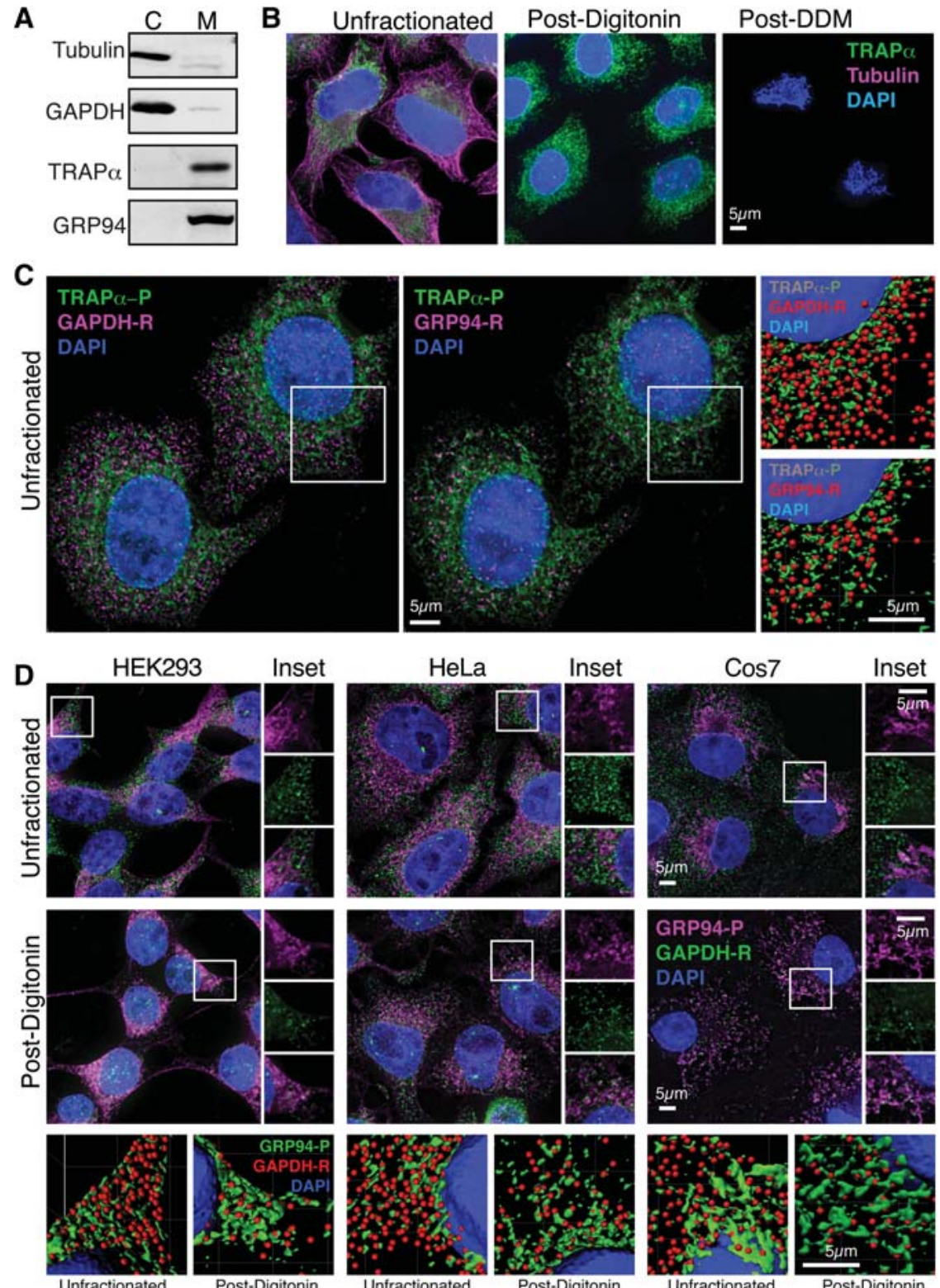

Unfractionated
Post-Digitonin

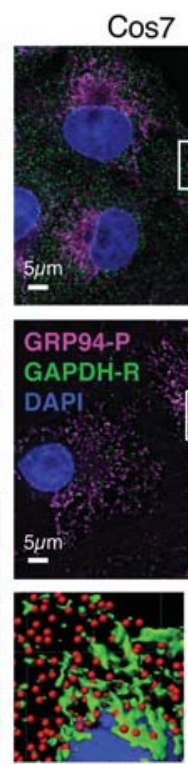

Unfractionated Inset

FIGURE 1. Single molecule RNA-FISH combined with cell fractionation reveals cytosolic protein-encoding mRNAs in proximal association with the ER. (A) Protein and RNA from the cytosol (C) and membrane-bound (M) fractions were extracted using TRIZOL reagent. Cytosolic (tubulin and GAPDH) and ER resident (TRAPa and GRP94) protein distributions were assessed by immunoblotting. (B) Immunostaining for cytosolic marker, tubulin (magenta), and ER marker, TRAPa (green), in unfractionated, digiton-extracted and DDM-extracted HeLa cells. $(C)$ GAPDH and GRP94 mRNAs (magenta) were visualized by smFISH along with immunostaining for an ER protein marker, TRAPa (green), in unfractionated HeLa cells. (D) GAPDH mRNA (green) and the ER marker, GRP94 (magenta), were visualized in unfractionated and digitonin-extracted cells by immunostaining and smRNA-FISH. Insets illustrate overlap in GAPDH mRNA and GRP94 staining patterns. Images represent maximum intensity projections, with $3 \mathrm{D}$ renderings of the de-convolved images presented in the bottom series of micrographs.

released upon digitonin treatment. The remaining pool displayed a distribution pattern that closely approximated the ER network. This conclusion is further detailed in the insets, which depict the individual channels and the merged images (Fig. 1D), and in 3D renderings of the de-convolved images, which reveal that the GAPDH mRNA pool remaining after digitonin extraction is almost entirely limited to ER and near-ER figures (Fig. $1 \mathrm{D}$, bottom panels). Importantly, this pool of GAPDH mRNAs was retained following extensive washing in digitonin and $0.5 \mathrm{M}$ salt-supplemented buffers, yet released upon addition of DDM (data not shown) (Fig. 4, below). These data corroborate past biochemical and molecular genetic studies of subcellular mRNA distributions and provide an additional line of evidence that $\mathrm{mRNA}_{\text {cyt }}$ can be localized to the ER.

A primary prediction of the de novo initiation model described above is that the subcellular distributions of $\mathrm{mRNA}_{\text {cyt }}$ and ribosomes would be similar and distinct from $\mathrm{mRNA}_{\mathrm{sec}}$. To test this prediction, we again used the sequential detergent fractionation method and examined the fractional distributions of representative mRNAs and ribosomes on the ER. As shown in Figure 2, qRT-PCR analysis of RNA subcellular distributions reveals that BiP- and GRP94-encoding mRNAs display a high enrichment and narrow distribution in the ER fraction and tRNA a high enrichment and narrow distribution in the cytosol fraction (Fig. 2). In contrast, $18 \mathrm{~S}$ rRNA and GAPDHand MAPK8-encoding mRNAs show similar and more variable subcellular distributions between the two compartments (Fig. 2). These data are consistent with the smRNA-FISH data reported in Figure 1 and demonstrate that the subcellular $\mathrm{mRNA}_{\text {cyt }}$ distributions parallel ribosomal distributions, rather than the expected cytosolic marker (tRNA). distinguish between cytosolic and ER-associated GAPDH mRNAs, combined smRNA-FISH/cell fractionation was performed, using three cell types, HEK293, HeLa, and Cos7 (Fig. 1D). In these experiments, two pools of GAPDH mRNA could be discerned. One pool, dispersed through the cytoplasm, was

\section{Cytosolic mRNA partitioning mirrors ribosome} partitioning: correlation with cell type-specific variations in subcellular ribosome distribution

We took several independent approaches to further test the hypothesis that translation initiation of $\mathrm{mRNA}_{\mathrm{cyt}}$ by 


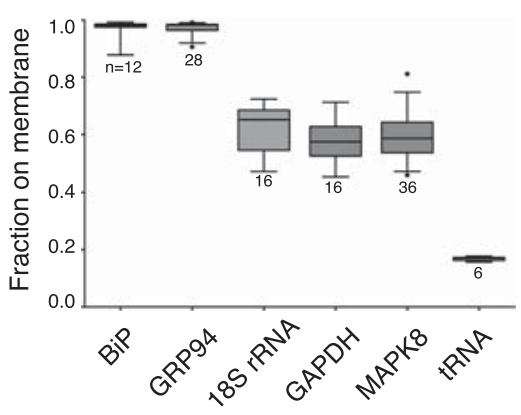

FIGURE 2. Subcellular distributions of cytosolic protein-encoding mRNAs correlate with ribosome distribution. RNA samples were analyzed by qRT-PCR to assess subcellular distributions of membranebound mRNAs (GRP94 and BiP), cytosolic mRNAs (GAPDH and MAPK8), tRNA, and 18S rRNA. A box-whisker plot representing the fraction of various mRNAs on the ER is shown. The average \pm SD is represented from multiple, independent experiments ( $n$ values as indicated).

membrane-bound ribosomes provides a mechanism for their ER localization. First, four different cell lines, HEK293, HeLa, HDF, and J558, with widely varying relative levels of ER, were fractionated in independent triplicate experiments and ribosome and GAPDH mRNA subcellular distributions determined by qRT-PCR. As shown in Figure 3A, a plot of the fraction of ER-bound ribosomes vs. the fraction of ER-bound GAPDH mRNA for all cell types examined revealed a high correlation between the two variables (Pearson coefficient $=0.9521$; Spearman coefficient $=0.9473$ ). Similarly, when paired fractionation values for rRNA and GAPDH mRNA from 16 independent fractionation experiments in HEK293 cells were plotted (Fig. 3B), a significant positive correlation was obtained. On the basis of these correlations, we conclude that the subcellular distribution of GAPDH-encoding mRNAs is linked to the subcellular ribosome distribution, which is, itself, determined by the amount of ER present in a given cell type and likely influenced by variables associated with tissue culture, such as growth status, passage number, and genetic drift.

To extend this analysis, we examined the subcellular mRNA distributions of several additional genes in HEK293 and 5558 cells. These two cell lines differ substantially in their subcellular ribosome distributions, with J558 cells displaying a much larger relative fraction of ER-associated ribosomes, as expected of a terminally differentiated professional secretory cell type. We performed a quantitative assessment of mRNA levels in the cytosol and ER compartments using commercially available 96-gene qPCR array sets, selected to include genes whose products encode prominent proteins of known function and subcellular distribution (Supplemental Table S1). Subcellular enrichment values for all mRNAs meeting threshold selection criteria in the GPCR analyses were calculated, and the results are represented as a histogram of the fraction of RNAs in the membrane compartment (Fig. 3C,D). As shown, the subcellular mRNA distribution in
HEK293 cells reflects the bimodal partitioning of RNAs between the cytosol and ER compartments (Fig. 3C, top panel). However, among the 37 cytosolic and nucleoplasmic proteinencoding RNAs, the lowest ER-enrichment value observed was only 0.34 (FOXM1). Thus, even within a limited sampling of mRNA subcellular distributions, cytosolic mRNAs are well represented on the ER. When the genes were subdivided into two cohorts based on the intracellular location of the encoded proteins (mRNA $_{\text {cyt }}$ for cytoplasmic and nucleoplasmic proteins; $\mathrm{mRNA}_{\mathrm{sec}}$ for secretory pathway cargo and endomembrane resident proteins), it is clear that while mRNA $_{\text {cyt }}$ display a slight preference for the cytoplasm (mean fractional enrichment of 0.43 on the ER), mRNA $_{\text {sec }}$ display a marked preference for the ER (mean enrichment of 0.78 on ER).

In J558 cells, in contrast, the mRNA populations show a marked shift toward enrichment in the ER compartment, and the bimodality observed in the mRNA population of HEK293 cells is not readily discernable (Fig. 3D, top panel). However, when the genes are grouped into $\mathrm{mRNA}_{\text {cyt }}$ and $\mathrm{mRNA}_{\mathrm{sec}}$, the bimodal distribution pattern again emerges. Here, the mRNA $_{\text {cyt }}$ have a higher mean ER enrichment as compared to HEK293 cells (mean population enrichment of 0.66 ) and the $\mathrm{mRNA}_{\mathrm{sec}}$ also a higher enrichment on the ER (mean enrichment of 0.84). Just as in HEK293 cells, certain cytosolic mRNAs, e.g., CAMKIIA (calcium/calmodulindependent protein kinase IIa) and PRKCD (protein kinase $\mathrm{C} \delta$ ) show a very high, noncanonical enrichment on the ER. Surprisingly, other $\mathrm{mRNA}_{\mathrm{sec}}$, such as ICOSL (inducible T cell costimulator), display noncanonical enrichment in the cytosol (Fig. 3D), the significance of which is currently unknown.

\section{ER-bound ribosomes translate $\mathrm{mRNA}_{\text {cyt }}$}

Having established that $\mathrm{mRNA}_{\text {cyt }}$ are localized to the ER membrane and that the fractional enrichment on the ER correlates with subcellular ribosome distributions, we next examined the ribosome-association status of these ER-associated transcripts. Here, digitonin-extracted cells were solubilized in DDM-supplemented buffers and mRNA-polysome distributions examined by both sucrose gradient sedimentation (Stephens and Nicchitta 2007; Jagannathan et al. 2011) and ribosome footprinting (Reid and Nicchitta 2012). For the former, cytosol and ER fractions from HeLa cells were fractionated on sucrose density gradients and mRNA distributions determined by Northern blotting (Fig. 4A). As depicted, the signal sequence-encoding mRNA, BiP, was highly enriched in the ER-heavy polysome fraction and largely absent from cytosolic polysomes. In contrast, mRNAs encoding the cytosolic proteins GAPDH and ATF4 were represented on both cytosolic and ER polysomes, and tRNAs were highly enriched in the cytosol fraction.

Though demonstrating that $\mathrm{mRNA}_{\text {cyt }}$ undergo translation on ER-bound ribosomes, the polysome profiling data 


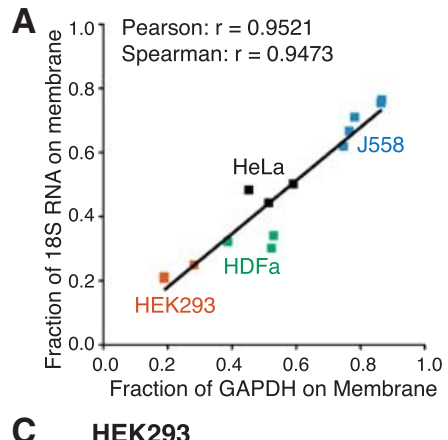

C
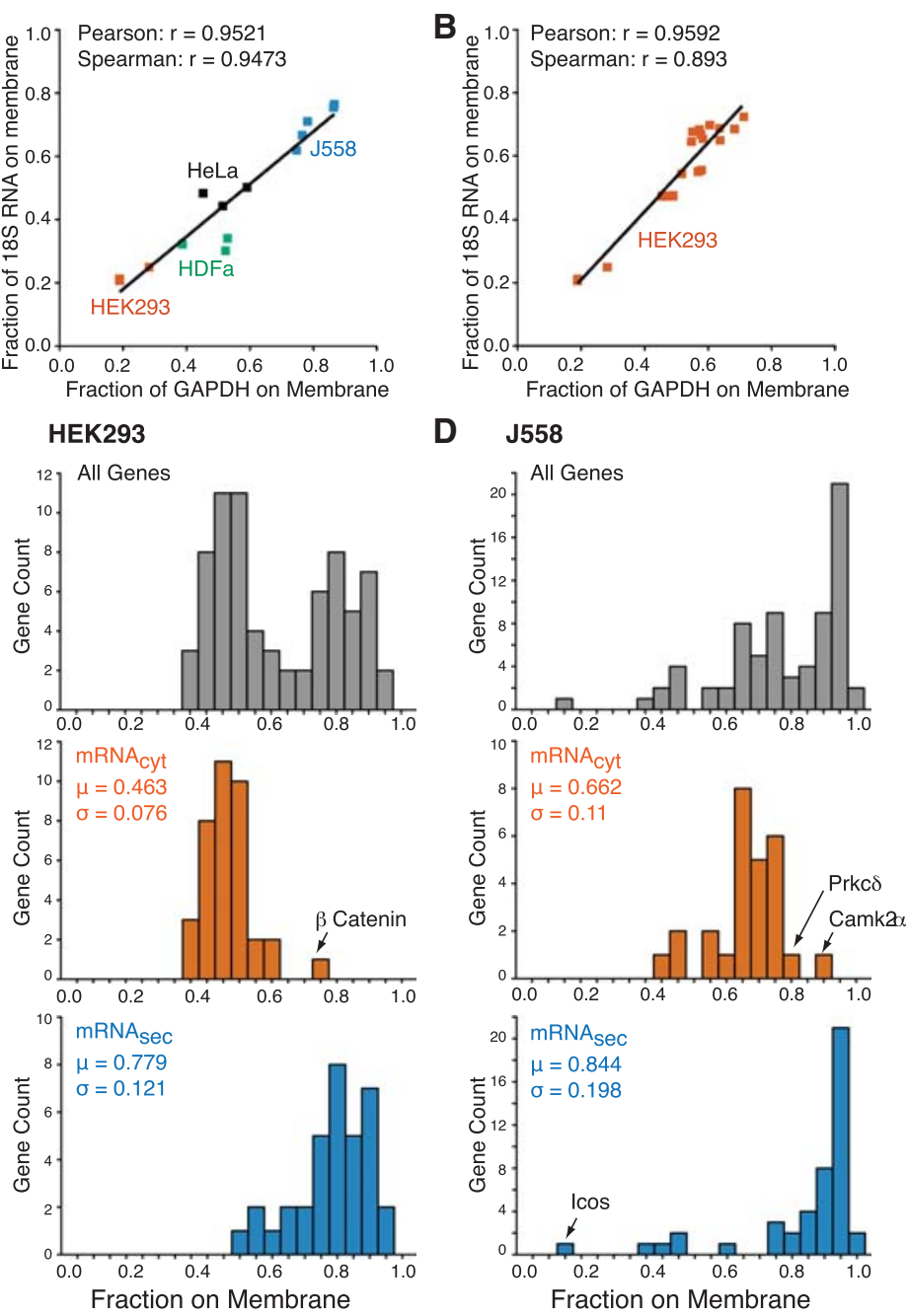

FIGURE 3. Correlation between paired measurements of the fraction of $18 \mathrm{~S}$ rRNA and GAPDH mRNA on the ER membrane. (A) Ribosome and GAPDH mRNA distributions were determined in the indicated cell lines and in 16 independent experiments conducted with HEK293 cells (B). qPCR array data comparing RNA distribution in cytosol and membrane fractions of HEK293 (C) and J558 (D) cells. Data are represented as a histogram of the fractional value of RNA in the membrane fraction for various cohorts of RNAs. RRNA $_{\text {cyt }}$ represent RNAs that encode cytosolic and nucleoplasmic proteins; $\mathrm{mRNA}_{\mathrm{sec}}$ include RNAs encoding secretory and membrane proteins as well as resident proteins of endomembrane organelles. $(\mu)$ Mean, $(\sigma)$ deviation.

depicted in Figure 4A do not distinguish between scenarios where cytosolic mRNAs are recruited to the ER at the stage of translation initiation or at a later stage of translation, e.g., via binding of polysomes to the ER membrane. This distinction is important as it may hold clues to the mechanism by which cytosolic mRNAs localize to the ER. To address this question, we analyzed RNA-Seq/ribosome footprinting data on cytosolic and ER-derived polysome fractions from HEK293 cells (Fig. 1B; Reid and Nicchitta 2012). Here, we reasoned that if ER-bound ribosomes are capable of de novo initiation, an enrichment of ribosome footprints centered about initiation codons would be expected. Alternatively, if translation initiation is restricted to cytosolic ribosomes, only those ribosomes should display a footprint corresponding to the start codon. The results of these analyses are shown in Figure $4 \mathrm{~B}$ and demonstrate that, at the mRNA transcriptome level, the footprinting profiles of the mRNA $_{\text {cyt }}$ fraction of ER-bound ribosomes are very similar to their cytosolic counterparts and include a robust enrichment at the start codon. This conclusion is further illustrated for a single mRNA (encoding the cytosolic protein ribosomal protein S5) (Fig. 4C) where the overall ribosome footprinting patterns of the cytosolic and ER-associated RSP5 mRNA populations are very similar and display a start codon read enrichment as well as similar natural translational pause sites throughout the open reading frame. These data demonstrate that cytosolic mRNAs can undergo translational initiation on the ER and are actively translated in both the cytosol and ER compartments.

\section{Translation initiation factor localization to the ER}

If de novo translation initiation occurs on the ER, it would be expected that eIF2, an essential initiation factor that mediates formation of the $43 \mathrm{~S}$ preinitiation complex, would itself be present on the ER. To test this prediction, HeLa cells were fractionated as described in Figure 1, the ER and cytosol fractions concentrated by TCA precipitation, and the subcellular distribution of the eIF2a subunit determined by immunoblot. As shown in Figure 5A, eIF2 $\alpha$ was found at approximately similar levels in both the cytosol and ER compartments. Intriguingly, in the semi-intact HeLa cell model, ER-associated eIF2 $\alpha$ behaved as a peripheral membrane protein; it was resistant to extraction with $0.2 \mathrm{M} \mathrm{KOAc}$, yet released upon addition of $0.4 \mathrm{M} \mathrm{KOAc}$ (Fig. 5B). Furthermore, ER-associated eIF2 $\alpha$ was released by addition of the nonionic detergent DDM in the presence of $0.2 \mathrm{M} \mathrm{KOAc}$, suggesting that it was either bound to an ER membrane protein(s) or to ER-associated polysomes. To distinguish between these two scenarios, the cytosol and detergent-soluble ER fractions were subjected to ultracentrifugation, to pellet ribosomes, and the distribution of eIF2 $\alpha$ between the ribosome-associated (pellet) and free (supernatant) fractions determined by immunoblot analysis. As shown in Figure 5C, for both the cytosol and 
A
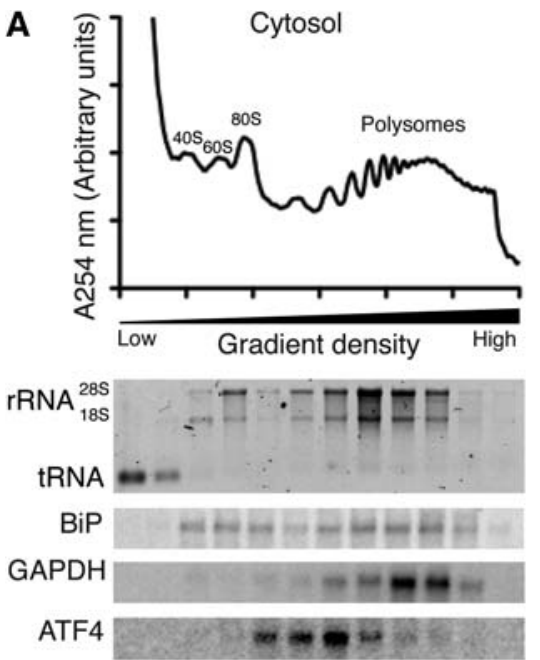

B All cytosolic protein-encoding genes

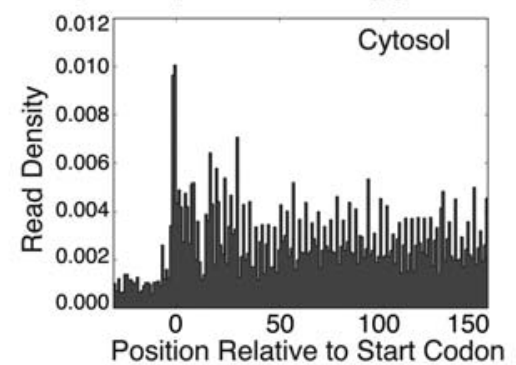

C RPS5

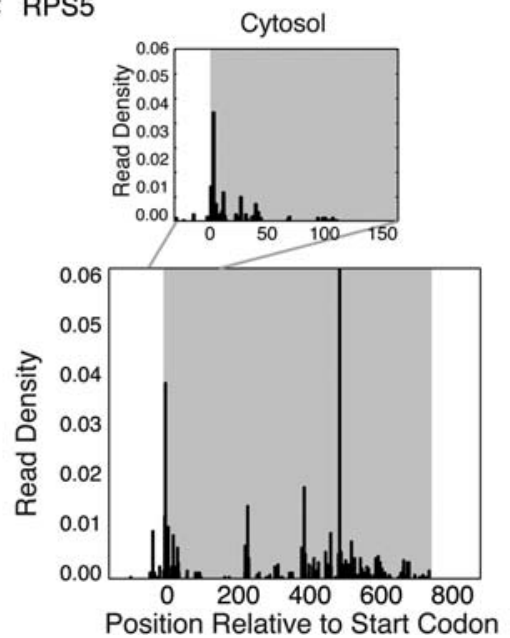

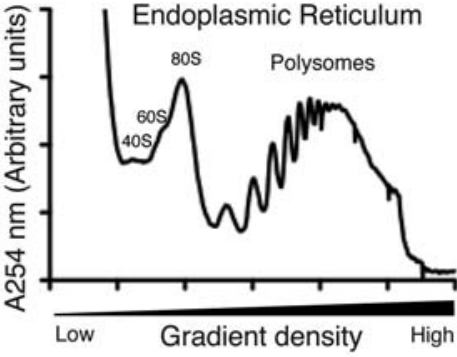
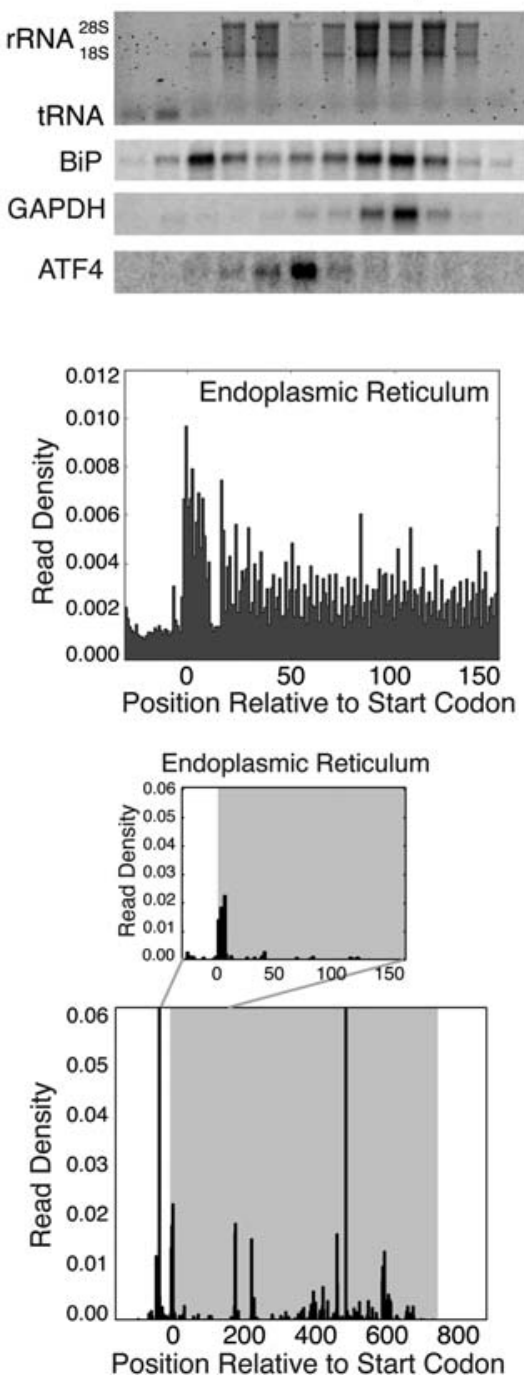

FIGURE 4. Cytosolic protein-encoding mRNAs undergo translation initiation and elongation on ER-bound ribosomes. (A) Cytosol and membrane-bound (endoplasmic reticulum) polysomes were resolved on $15 \%-50 \%$ sucrose gradients. The position of the ribosomes in the gradient was assessed by UV spectrometry $\left(\mathrm{A}_{254} \mathrm{~nm}\right)$. Total RNA extracted from the sucrose gradient fractions was analyzed by denaturing agarose gel electrophoresis followed by Northern blot for GRP94 and GAPDH mRNAs. The RNA integrity is demonstrated by methylene blue staining of rRNA. (B) Distribution of ribosome footprints on all cytosolic protein-encoding mRNAs and a specific mRNA, RPS5 $(C)$ in the cytosol and ER compartments (Reid and Nicchitta 2012).

detergent-soluble ER fractions, eIF2 $\alpha$ was recovered entirely in the supernatant. These data indicate that a substantial fraction of eIF $2 \alpha$ is localized to the ER via binding interactions

with an as yet unknown component(s) of the ER membrane. We presume that this fraction participates in ER-associated de novo translation initiation, though this has not been established.

\section{A stochastic translation initiation model for the subcellular mRNA partitioning of cytosolic protein- encoding mRNAs}

The data presented above demonstrate that cytosolic protein-encoding RNAs can be localized to the ER (Fig. 1), that the extent of their ER-targeting correlates positively with the amount of ER-bound ribosomes present in a given cell population at a given time (Figs. 2, 3), and that $\mathrm{mRNA}_{\text {cyt }}$ both initiate and sustain translation on ER-bound ribosomes (Figs. 4, $5)$. These findings are summarized in the following model depicting known and proposed ribosome/mRNA translation and trafficking dynamics (Fig. 6).

In the case of cytosolic protein-encoding mRNAs, translation could initiate on either free or bound ribosomes (Ribo $_{\mathrm{Cyt}}$ and Ribo ${ }_{\mathrm{ER}}$, respectively). A free ribosome translating an $\mathrm{mRNA}_{\text {cyt }}$ would be expected to remain in the cytosol, whereas a membrane-bound ribosome translating an $\mathrm{mRNA}_{\text {cyt }}$ may either remain ER-associated or dissociate from the ER via a mechanism previously termed elongation coupled ribosome release (ECRR) (Nicchitta et al. 2005). In the case of signal sequence-encoding mRNAs, translation could also initiate on free or bound ribosomes. For free ribosomes, the SRP pathway would recruit the mRNA/ribosome/nascent chain complex to the ER, as has been well established (Walter and Lingappa 1986; Blobel 2000). For bound ribosomes, translation and protein translocation can proceed without a requisite SRP targeting step (Potter and Nicchitta 2000; Potter et al. 2001). Through these distinct trafficking avenues, the model accounts for the broad presence of $\mathrm{mRNA}_{\text {cyt }}$ on the ER, the high ER enrichments of $\mathrm{mRNA}_{\mathrm{sec}}$ on the ER, and provides a rationale for the high correlation observed between the ribosome distribution and $\mathrm{mRNA}_{\mathrm{cyt}}$ distribution in cells. Further studies to test this model are currently under way. 


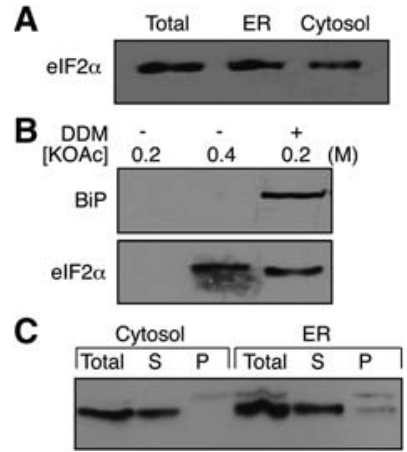

FIGURE 5. eIF2 $\alpha$ is partitioned between the cytosol and ER. (A) HeLa cells were fractionated using sequential detergent solubilization, the cytosol and ER fractions concentrated by TCA precipitation, and eIF2 $\alpha$ subcellular distributions determined by immunoblot analysis. (B) Cytosolic contents of HeLa cells were released using digitonin as per above. Semi-intact cells were subsequently treated with 200 or 400 $\mathrm{mM}$ KOAc in cytosol buffer to strip peripheral membrane proteins in the absence or presence of $2 \%$ DDM, and eIF2 $\alpha$ determined by immunoblot analysis. Paired analyses with a BiP antibody are included as a measure of ER integrity. (C) HeLa cells were fractionated as described above and cytosol and ER fractions obtained. One half of each sample was reserved as the "total," and the remaining half was subjected to ultracentrifugation to obtain the ribosome fraction. Supernatant fractions were concentrated by TCA precipitation, and the pellet (ribosome) fraction was directly resuspended in SDS-PAGE sample buffer. Cell equivalent volumes of each fraction were analyzed by SDS-PAGE and immunoblot against eIF2 $\alpha$.

\section{DISCUSSION}

\section{Assessing subcellular RNA distribution by cell fractionation}

Cellular protein synthesis is a function shared by two populations of ribosomes, namely, cytosolic and ER-bound. While cytosolic and nucleoplasmic proteins are predominately synthesized on cytosolic ribosomes, secretory pathway proteins are synthesized on ER-bound ribosomes. This binary mechanism ensures the correct subcellular localization of proteins and directs the segregation of RNAs between the two populations of ribosomes. Typically, subcellular mRNA distributions are determined by assessing the amount of RNA present in cytosol and ER compartments of fractionated cells (Mueckler and Pitot 1981; Lerner et al. 2003; Diehn et al. 2006; Chen et al. 2011; Reid and Nicchitta 2012). However, and although the various cell disruption approaches yield highly enriched subfractions, as determined by marker protein distributions, cytosolic protein-encoding RNAs are found to be broadly represented on the ER. This could either mean that cell fractionation is an unreliable method for assessing subcellular RNA distributions or that subcellular RNA distributions are regulated by multiple mechanisms.

Here, using single molecule RNA fluorescence in situ hybridization and cell fractionation, we have demonstrated that a candidate RNA with an expected cytosolic distribution, GAPDH, does, indeed, reside in close proximity to the ER membrane and, by virtue of its extractability in detergent, can be translated on ER-bound ribosomes. We have also shown that the fraction of GAPDH on the membrane shows a significant positive correlation with the fraction of ribosomes that are membrane-bound. This correlation is also seen at an mRNA population level, particularly when comparing cytosolic RNA distributions in cell lines with quite different amounts of membrane-bound mRNAs; cells with a higher fraction of ER-bound ribosomes also display a higher fraction of ER-bound cytosolic mRNA. Thus, it appears that the discordance between the expected and observed distribution of cytosolic mRNAs is not due to a deficiency in the method of assessment but rather reflects gaps in our understanding of how mRNAs engage ER-bound ribosomes.

\section{Does all translation begin in the cytoplasm?}

The expectation that cytosolic mRNAs and ER mRNAs are segregated from one another stems from the generally held view that translation begins (uniquely) in the cytoplasm. In this view, mRNAs that encode a protein destined for the secretory pathway are positively selected for localization to the ER, and cytosolic mRNAs remain cytosolic by default. Without question, in vitro studies demonstrate that, when translation of an mRNA encoding a secretory protein is initiated on free ribosomes, SRP efficiently directs the trafficking of the mRNA/ribosome/nascent chain to the ER (Walter and Blobel 1981a,b; Walter et al. 1981). However, in the complementary experiment, where rough microsomes

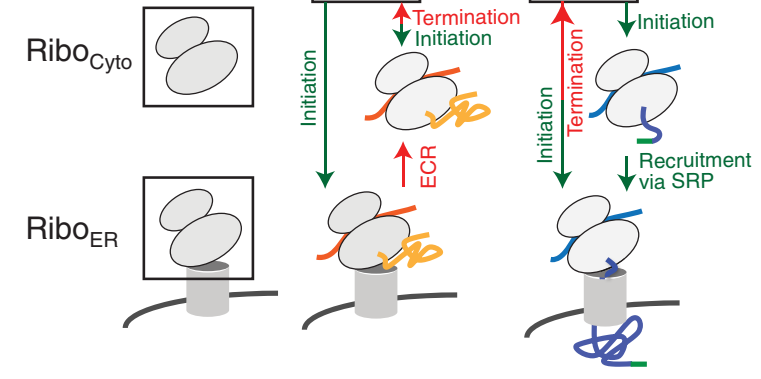

FIGURE 6. Model depicting pathways for regulating ribosome and mRNA partitioning between the cytosol and ER compartments. mRNA $_{\text {cyt }}$ is shown in orange, cytosolic protein in yellow, mRNA $A_{\text {sec }}$ in light blue, and a secretory pathway protein in dark blue. The signal peptide is colored green. Arrows represent association and dissociation reactions that direct subcellular ribosome and mRNA partitioning. For $\mathrm{mRNA}_{\text {cyto }}$, mRNAs are depicted as undergoing translation initiation on either cytosolic or ER-bound ribosomes. For the latter, the mRNA could remain ER-associated, or the translating ribosome could dissociate from the ER via elongation-coupled ribosome release (ECRR). For mRNA $_{\text {sec }}$, mRNAs could also undergo translation initiation on either cytosolic or ER-bound ribosomes. In the former case, the mRNA would be localized to the ER via the SRP pathway. In the latter, the mRNA would remain ER-associated. 
are incubated with a ribosome-depleted reticulocyte lysate, so that initiation is restricted to membrane-bound ribosomes, the bound ribosomes were found to be competent for de novo translation initiation of both cytosolic- and secretory protein-encoding mRNAs alike (Potter and Nicchitta 2000, 2002). These results, combined with the findings from recent ribosome footprinting studies that cytosolic protein-encoding mRNAs undergo translation initiation on ER-bound ribosomes (Reid and Nicchitta 2012), lead us to suggest that as all newly transcribed mRNAs exit the nucleus, their initial (pioneer) translation could occur on either free or bound ribosomes. Individual mRNAs may show a preference for one or the other compartment, should they encode specific localization sequences. In the absence of such a preference, however, the subcelllular site of initiation appears to be largely dependent on the relative availability of ribosomes in the cytosol versus the ER (Fig. 6).

\section{A role for the ER in global proteome expression}

mRNAs that are bound to the ER membrane are subjected to modes of post-transcriptional regulation that are often distinct from that encountered by cytosolic mRNAs. For example, ER-bound mRNAs are translated more efficiently (Stephens and Nicchitta 2008; Reid and Nicchitta 2012), and so the contribution to gene expression from an ER-bound population of a cytosolic protein-encoding mRNA may exceed that of its cytosolic counterpart. Because essentially the entire mRNA transcriptome is found on the ER, it is of value to revisit current models regarding roles for the ER in proteome expression and now consider the ER as a primary contributor to global gene expression. Thus, in addition to a dedicated role in secretory and membrane protein biogenesis, the ER also contributes substantially to the translation of all mRNAs. Indeed, in a limited set of cases, cytosolic proteinencoding mRNAs may be highly partitioned to the ER, and the near entirety of their expression may occur on the ER (Diehn et al. 2006; Reid and Nicchitta 2012). The biological significance of this phenomenon remains to be established and will require identification of the mechanisms guiding such noncanonical localization and their role(s) in the regulation of protein expression and function. These findings do, however, illustrate that the subcellular architecture of mRNA translation is complex and likely subject to diverse and mRNA-selective post-transcriptional regulatory controls. Integrating these findings into the broad biology of cell structure and function promises to reveal new paradigms for both mRNA localization and post-transcriptional gene regulation.

\section{MATERIALS AND METHODS}

\section{Reagents and cell culture}

All reagents were purchased from Sigma unless otherwise indicated. All cell lines were maintained at $37^{\circ} \mathrm{C}$ and $5 \% \mathrm{CO}_{2}$. HeLa and
HEK293 cells were cultured in Dulbecco's modified Eagle's medium (DMEM; Mediatech), supplemented with $10 \%$ fetal bovine serum (FBS; Life Technologies). HDFa cells were maintained in M106 medium (Life Technologies) containing low-serum growth supplement (Life Technologies). J558 cells were maintained in DMEM supplemented with $10 \%$ horse serum.

\section{Detergent extraction}

Detergent fractionations were performed on ice with cells at 75\%$90 \%$ confluence. Cells were incubated on ice for $20 \mathrm{~min}$ in PBS/ $\mathrm{MgCl}_{2}$ and $50 \mu \mathrm{g} / \mathrm{mL}$ cycloheximide (Sigma) to de-polymerize microtubules while stabilizing polyribosomes. Cell monolayers were extracted for $10 \mathrm{~min}$ with a digitonin-containing permeabilization buffer $(0.015 \%$ digitonin [Calbiochem] in $110 \mathrm{mM} \mathrm{KCl}, 25 \mathrm{mM}$ K-HEPES at pH 7.4, $1 \mathrm{mM} \mathrm{MgCl}_{2}, 0.1 \mathrm{mM}$ EGTA, 40 units/mL RNAseOUT (Life Technologies), and $1 \mathrm{mM}$ DTT). The resulting cytosol fraction was recovered, and the cells were rinsed in a buffer of identical composition containing $0.004 \%$ digitonin. The wash fraction was combined with the cytosolic extract. The digitonin-extracted cells were then lysed by addition of $2 \%$ dodecylmaltoside (DDM) in $200 \mathrm{mM} \mathrm{KCl}, 25 \mathrm{mM} \mathrm{K}$-HEPES at $\mathrm{pH} 7.4,10 \mathrm{mM} \mathrm{MgCl}_{2}, 40$ units/mL RNAseOUT, and $2 \mathrm{mM}$ DTT for $10 \mathrm{~min}$ (Stephens et al. 2008; Jagannathan et al. 2011).

\section{RNA extraction and quality assessment}

Three biological replicates for each of the samples were generated, and RNA was extracted by the standard acid guanidinium thiocyanate (GT)-phenol-chloroform extraction procedure (Chomczynski and Sacchi 2006), using GlycoBlue (Ambion) as a carrier to ensure quantitative precipitation of the RNA. The samples were then treated with Turbo DNAse (Ambion) and RNA recovered using an RNeasy mini kit (Qiagen) or by re-extraction in GT:phenol. RNA samples were quantified by UV spectrometry (NanoDrop Tech.). In the case of StellArray analysis, quality was also assessed by an Agilent 2100 Bioanalyzer with the RNA 6000 Nano Labchip kit. Only those samples with an RNA integrity number (RIN) in the range of 9.0-10 were used for subsequent analysis.

\section{Quantitative reverse transcriptase (RT)-PCR}

Cell-equivalent amounts of RNA were used to synthesize cDNA using M-MLV reverse transcriptase (Promega) and random hexamers as primers (Roche). Quantitative RT-PCR was performed on a 7900HT Sequence Detection System (Applied Biosystems) using Power SYBR Green PCR Mastermix (Applied Biosystems). Except for $18 \mathrm{~S}$ rRNA, all primers were designed to be intron-spanning using the Primer3-based ProbeFinder software (Roche). The primer sequences are as follows:

18SrRNA (F: CACGGGAAACCTCACCCGGC; R: CGGGTGGCTG AACGCCACTT); GRP94 (F: CTGGAAATGAGGAACTAACAG TCA; R:TCTTCTCTGGTCATTCCTACACC); BiP (F: CAACCAACTGTTACAATCAAGGTC; R: CAAAGGTGACTTCAATCTGTGG);

GAPDH (F: AGCCACATCGCTCAGACAC; R: GCCCAATACGAC CAAATCC); MAPK8 (F: GGGCAGCCCTCTCCTTTA; R: CAT TGACAGACGACGATGATG); B2M (F:TTCTGCCCTGGAGG 
CTATC; R: TCAGGAAATTTGACTTTCCATTCTCAGGAAAT TTGACTTTCCATTC);

tRNA (F: GTCAGGATGGCCGAGTGGTCTAAG; R: CCACGCC TCCATACGGAGAACCAGAAGACCC).

At least three biological replicates of all RNA samples were independently processed for every experimental condition, and the qRTPCR data plotted represent the mean and standard error from the replicate experiments.

\section{StellArray qPCR array and data analysis}

To assess RNA partitioning at a subgenomic level, a StellArray qPCR Array (Lonza) consisting of pre-deposited primers for 96 genes was used. RNA extracted from three biological replicates was reversetranscribed as described earlier. The $\mathrm{qPCR}$ reactions were performed using the cycling conditions recommended by the manufacturer. The threshold cycle was manually set to the same value for all the qPCR arrays, and the data were analyzed either by the ddCt method or by the Global Pattern Recognition (GPR) program (Bar Harbor Technology). The GPR program selects a normalizer gene from a set of invariant genes in the data set, thus eliminating the need for identifying a normalizer gene a priori.

\section{Protein extraction, SDS-PAGE, and immunoblotting}

Proteins in cell extracts were concentrated by TCA precipitation. The protein pellets were resuspended in a sample buffer containing $0.5 \mathrm{M}$ Tris base and 5\% SDS. Protein concentrations were determined by BCA assay (Pierce). Either cell-equivalent or equal amounts of protein samples were analyzed by reducing SDSPAGE. Immunoblotting was conducted by standard protocols using antibodies against tubulin, GAPDH, GRP94, and TRAPa.

\section{Immunofluorescence staining}

HeLa cell monolayers were fixed in $10 \%$ formalin for $10 \mathrm{~min}$ at room temperature. After blocking with $1 \%$ bovine serum albumin (BSA) in PBS, the cells were incubated in a primary antibody solution for $1 \mathrm{~h}$, extensively washed and incubated in Alexafluor(AF) 488 or AF550-labeled secondary antibody solution (Life Technologies) for $45 \mathrm{~min}$. Cell monolayers were again washed and the nuclei stained using DAPI. The cells were then mounted in a mounting solution containing $0.4 \%$ glucose in PBS, and supplemented with glucose oxidase and catalase for imaging.

\section{Single molecule RNA fluorescence in situ hybridization}

Single RNA molecules were visualized using Stellaris FISH probes (Biosearch Technologies) as per the manufacturer's instructions. All reagents were made up in DEPC-treated deionized water, and standard precautions were taken to prevent RNA degradation. Briefly, HeLa cell monolayers were fixed in $10 \%$ formalin in PBS for $10 \mathrm{~min}$ at room temperature. After three 5-min washes in PBS, the cells were permeabilized overnight in cold $70 \%$ ethanol. The cells were equilibrated in $2 \times$ saline-sodium citrate (SSC) buffer and hybridized with a fluorescently labeled oligonucleotide probe mixture for $4-12 \mathrm{~h}$ at $37^{\circ} \mathrm{C}$. GAPDH probes were labeled with Quasar 570 and GRP94 probes labeled with Quasar 670 fluorophores. Excess probes were washed by two 30 -min incubations in $2 \times$ SSC containing $10 \%$ formamide at $37^{\circ} \mathrm{C}$. The cells were then washed in $2 \times \mathrm{SSC}$ containing DAPI and equilibrated in GLOX buffer $(0.4 \%$ glucose in $2 \times$ SSC and $1 \mathrm{mM}$ Tris $\mathrm{HCl}, \mathrm{pH}$ 7.4) before mounting in GLOX buffer supplemented with glucose oxidase and catalase. For costaining protein and RNA, cells were first blocked in RNAse-free UltraPure BSA (Ambion) and proteins immunolabeled with fluorescent antibodies under RNAse-free conditions. The cells were then fixed a second time in ice-cold methanol for $5 \mathrm{~min}$, and then smRNA-FISH was performed as described above.

\section{Fluorescence imaging and analysis}

All imaging was performed on a DeltaVision Elite de-convolution microscope (Applied Precision) with the $100 \times /$ NA 1.40 oil immersion objective (UPlanSApo 100XO; Olympus), polychroic quad filter set to image fluorophores in DAPI, FITC, TRITC, and CY5 channels (peak/bandwidth/nm: EX: 350/50, 402/15, 490/20, 555/ 25, 645/30; EM: 446/20, 520/15, 592/20, 675/35/40), and a high-resolution CCD camera (CoolSNAP HQ2; Photometrics). Images were acquired as Z-stacks at $0.2-\mu \mathrm{m}$ intervals with identical exposure settings across the samples for a given probe. The data were de-convolved using the SoftWoRx program (Applied Precision) and processed further on ImageJ/FIJI software and Adobe Photoshop (Adobe Systems) to render maximum intensity projections, merge channels, and pseudocolor as needed. Only linear changes were performed to the brightness/contrast values of the images, as required, and such changes were applied uniformly across all images in a given experiment. For smRNA-FISH data, the brightness/contrast was adjusted to ensure optimal visibility of the RNA molecules, while not altering the number of molecules in a given sample. De-convolved image stacks were 3D-rendered using Imaris (Bitplane) with the following settings: The nucleus (DAPI staining) and ER network (detected by immunostaining) were rendered in $3 \mathrm{D}$ as "volume" objects with thresholding by background subtraction and smoothed with a surface area detail level of $0.134 \mu \mathrm{m}$ for the nucleus and $0.1 \mu \mathrm{m}$ for the ER. The RNA molecules (detected by smFISH) were rendered as "spot" objects due to their uniform and compact nature using automatic thresholding. The composite 3D image was captured using the "snapshot" option of Imaris. The final figures were assembled on Adobe Illustrator (Adobe Systems).

\section{Statistical analysis}

Mean and standard error values were calculated in Microsoft Excel, and all graphs were plotted using GraphPad Prism (GraphPad Software, Inc.). Pearson's and Spearman's correlation coefficients were calculated using GraphPad Prism.

\section{SUPPLEMENTAL MATERIAL}

Supplemental material is available for this article.

\section{ACKNOWLEDGMENTS}

The authors thank Sam Johnson, Director of the Duke Light Microscopy Core facility, for his extensive support and advice. We 
thank current and former members of the Nicchitta laboratory for their thoughts and discussions that led to this work. This work was supported by grants from the National Institute of General Medical Sciences of the NIH (GM101533 to C.V.N.), a bridge-funding award from the Duke University School of Medicine, and a shared instrumentation grant from the NSF (1S10RR027528-01).

Received March 25, 2014; accepted July 25, 2014.

\section{REFERENCES}

Akopian D, Shen K, Zhang X, Shan SO. 2013. Signal recognition particle: an essential protein-targeting machine. Annu Rev Biochem 82: 693-721.

Blobel G. 2000. Protein targeting (Nobel lecture). Chembiochem 1: 86-102.

Chen Q, Jagannathan S, Reid DW, Zheng T, Nicchitta CV. 2011. Hierarchical regulation of mRNA partitioning between the cytoplasm and the endoplasmic reticulum of mammalian cells. Mol Biol Cell 22: 2646-2658.

Chomczynski P, Sacchi N. 2006. The single-step method of RNA isolation by acid guanidinium thiocyanate-phenol-chloroform extraction: twenty-something years on. Nat Protoc 1: 581-585.

Cui XA, Zhang H, Palazzo AF. 2012. p180 promotes the ribosome-independent localization of a subset of mRNA to the endoplasmic reticulum. PLoS Biol 10: e1001336.

del Alamo M, Hogan DJ, Pechmann S, Albanese V, Brown PO, Frydman J. 2011. Defining the specificity of cotranslationally acting chaperones by systematic analysis of mRNAs associated with ribosome-nascent chain complexes. PLoS Biol 9: e1001100.

Diehn M, Eisen MB, Botstein D, Brown PO. 2000. Large-scale identification of secreted and membrane-associated gene products using DNA microarrays. Nat Genet 25: 58-62.

Diehn M, Bhattacharya R, Botstein D, Brown PO. 2006. Genome-scale identification of membrane-associated human mRNAs. PLoS Genet 2: e11.

Eliscovich C, Buxbaum AR, Katz ZB, Singer RH. 2013. mRNA on the move: the road to its biological destiny. J Biol Chem 288: 2036120368.

Jagannathan S, Nwosu C, Nicchitta CV. 2011. Analyzing mRNA localization to the endoplasmic reticulum via cell fractionation. Methods Mol Biol 714: 301-321.

Kraut-Cohen J, Afanasieva E, Haim-Vilmovsky L, Slobodin B, Yosef I, Bibi E, Gerst JE. 2013. Translation- and SRP-independent mRNA targeting to the endoplasmic reticulum in the yeast Saccharomyces cerevisiae. Mol Biol Cell 24: 3069-3084.

Lerner RS, Seiser RM, Zheng T, Lager PJ, Reedy MC, Keene JD, Nicchitta CV. 2003. Partitioning and translation of mRNAs encoding soluble proteins on membrane-bound ribosomes. RNA 9: 1123-1137.

Lingappa VR, Blobel G. 1980. Early events in the biosynthesis of secretory and membrane proteins: the signal hypothesis. Recent Prog Horm Res 36: 451-475.

Loya A, Pnueli L, Yosefzon Y, Wexler Y, Ziv-Ukelson M, Arava Y. 2008. The 3'-UTR mediates the cellular localization of an mRNA encoding a short plasma membrane protein. RNA 14: 1352-1365.

Martin KC, Ephrussi A. 2009. mRNA localization: gene expression in the spatial dimension. Cell 136: 719-730.
Mechler B, Rabbitts TH. 1981. Membrane-bound ribosomes of myeloma cells. IV. mRNA complexity of free and membrane-bound polysomes. I Cell Biol 88: 29-36.

Mueckler MM, Pitot HC. 1981. Structure and function of rat liver polysome populations. I. Complexity, frequency distribution, and degree of uniqueness of free and membrane-bound polysomal polyadenylate-containing RNA populations. J Cell Biol 90: 495-506.

Nicchitta CV, Lerner RS, Stephens SB, Dodd RD, Pyhtila B. 2005. Pathways for compartmentalizing protein synthesis in eukaryotic cells: the template-partitioning model. Biochem Cell Biol 83: 687-695.

Potter MD, Nicchitta CV. 2000. Regulation of ribosome detachment from the mammalian endoplasmic reticulum membrane. J Biol Chem 275: 33828-33835.

Potter MD, Nicchitta CV. 2002. Endoplasmic reticulum-bound ribosomes reside in stable association with the translocon following termination of protein synthesis. J Biol Chem 277: 23314-23320.

Potter MD, Seiser RM, Nicchitta CV. 2001. Ribosome exchange revisited: a mechanism for translation-coupled ribosome detachment from the ER membrane. Trends Cell Biol 11: 112-115.

Pyhtila B, Zheng T, Lager PJ, Keene JD, Reedy MC, Nicchitta CV. 2008. Signal sequence- and translation-independent mRNA localization to the endoplasmic reticulum. RNA 14: 445-453.

Raj A, van den Bogaard P, Rifkin SA, van Oudenaarden A, Tyagi S. 2008. Imaging individual mRNA molecules using multiple singly labeled probes. Nat Methods 5: 877-879.

Reid DW, Nicchitta CV. 2012. Primary role for endoplasmic reticulumbound ribosomes in cellular translation identified by ribosome profiling. J Biol Chem 287: 5518-5527.

Stephens SB, Nicchitta CV. 2007. In vitro and tissue culture methods for analysis of translation initiation on the endoplasmic reticulum. Methods Enzymol 431: 47-60.

Stephens SB, Nicchitta CV. 2008. Divergent regulation of protein synthesis in the cytosol and endoplasmic reticulum compartments of mammalian cells. Mol Biol Cell 19: 623-632.

Stephens SB, Dodd RD, Lerner RS, Pyhtila BM, Nicchitta CV. 2008. Analysis of mRNA partitioning between the cytosol and endoplasmic reticulum compartments of mammalian cells. Methods Mol Biol 419: 197-214.

Walter P, Blobel G. 1981a. Translocation of proteins across the endoplasmic reticulum III. Signal recognition protein (SRP) causes signal sequence-dependent and site-specific arrest of chain elongation that is released by microsomal membranes. J Cell Biol 91: $557-561$.

Walter P, Blobel G. 1981b. Translocation of proteins across the endoplasmic reticulum. II. Signal recognition protein (SRP) mediates the selective binding to microsomal membranes of in-vitro-assembled polysomes synthesizing secretory protein. J Cell Biol 91: 551-556.

Walter P, Johnson AE. 1994. Signal sequence recognition and protein targeting to the endoplasmic reticulum membrane. Annu Rev Cell Biol 10: 87-119.

Walter P, Lingappa VR. 1986. Mechanism of protein translocation across the endoplasmic reticulum membrane. Annu Rev Cell Biol 2: 499-516.

Walter P, Ibrahimi I, Blobel G. 1981. Translocation of proteins across the endoplasmic reticulum. I. Signal recognition protein (SRP) binds to in-vitro-assembled polysomes synthesizing secretory protein. J Cell Biol 91: 545-550. 


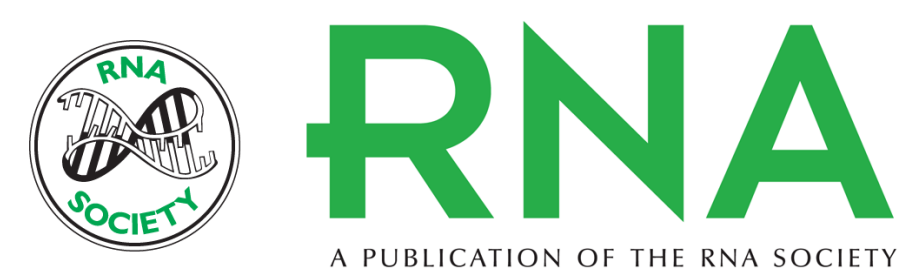

\title{
De novo translation initiation on membrane-bound ribosomes as a mechanism for localization of cytosolic protein mRNAs to the endoplasmic reticulum
}

\author{
Sujatha Jagannathan, David W. Reid, Amanda H. Cox, et al.
}

RNA 2014 20: 1489-1498 originally published online August 20, 2014

Access the most recent version at doi:10.1261/rna.045526.114

\section{Supplemental http://rnajournal.cshlp.org/content/suppl/2014/08/05/rna.045526.114.DC1 \\ Material}

References This article cites 32 articles, 15 of which can be accessed free at: http://rnajournal.cshlp.org/content/20/10/1489.full.html\#ref-list-1

Creative This article is distributed exclusively by the RNA Society for the first 12 months after the Commons full-issue publication date (see http://rnajournal.cshlp.org/site/misc/terms.xhtml). After 12 License months, it is available under a Creative Commons License (Attribution-NonCommercial 4.0 International), as described at http://creativecommons.org/licenses/by-nc/4.0/.

Email Alerting Receive free email alerts when new articles cite this article - sign up in the box at the top Service right corner of the article or click here. 